\title{
Chronic neurological disease as an independent risk factor for death in severe COVID-19 cases
}

\author{
Elena Caires Silveira
}

Federal University of Bahia, Multidisciplinary Institute of Health, Vitória da Conquista, Bahia, Brazil

\begin{abstract}
COVID-19 is a multisystemic disease with a wild severity range, being some chronic diseases risk factors for unfavorable evolution. It has been suggested are chronic neurological diseases are associated to higher mortality in COVID-19 patients; such association however, has not been described enough. In this context, this study seeks to evaluate whether the presence of previous chronic neurological disease is a factor associated with higher mortality in hospitalized severe cases of COVID-19. For this, the association between those variables was investigated in 87,871 patients through univariate (risk ratio and $X 2$ test) and multivariate (Poisson regression) analysis. It was found that the mortality rate for patients presenting chronic neurological disease was $23 \%$ higher (), this being an independent and statistically significant association $(R R=1.23,95 \% \mathrm{Cl}=1.2-1.3 ; p$-value $<0.001)$. Therefore, more studies are needed to better characterize this association.
\end{abstract}

Keywords: nervous system diseases, COVID-19, chronic disease, SARS-CoV-2

\section{INTRODUCTION}

Coronavirus disease 2019 (COVID-19), caused by SARS-CoV-2, is a multisystemic disease with a wild severity range, with cases varying from asymptomatic to cases evolving to death. In this context, it is known that advanced age, obesity, and some chronic diseases (such as hypertension, diabetes, COPD, and cancer) constitute risk factors for unfavorable evolution (1-3).

The high mortality of this disease determines along with the high demand for prolonged hospitalization, invasive procedures and intensive monitoring in severe cases - the high health and socioeconomic burden caused by the pandemic. Therefore, it is imperative to thoroughly investigate the factors associated with higher death rates from COVID-19.

Among the previous chronic conditions for which a relationship with negative outcomes in pa- tients infected with SARS-COV-2 has been suggested are chronic neurological diseases, whose association with this viral infection, however, has not been described enough (4). It has been established that COVID-19 may lead to neurological symptoms and comorbidities (5-7), but it is not yet well established whether previous neurological conditions may increase the risk of a worse disease course. Thus, in addition to the indirect health impairment of neurological patients related to health care burden and social constraints (8), it is sought to know if COVID-19 may present with greater severity in these patients.

In line with this objective, the present study seeks, from the evaluation of the risk ratio between patients with and without chronic neurological disease, to understand whether the presence of previous neurological disease is a factor associated with higher mortality in hospitalized severe cases of COVID-19. 


\section{METHODS}

This is a retrospective cross-sectional study conducted from patient level data of individuals hospitalized for COVID-19 in the period from January $3^{\text {th }}, 2021$ to $29^{\text {th }}$ May, 2021 presenting with flu-like syndrome and at least one of the following: (i) dyspnea/breathing discomfort; (ii) persistent chest pressure; (iii) oxygen saturation less than $95 \%$ on room air; (iv) bluish coloration of the lips or face (9). Data were collected from the SARS 2021 Severe Acute Respiratory Syndrome Database, publicly available, in the form of anonymized data, by the Brazilian Ministry of Health and fed by notifications of cases consistent with the description presented (10). It was considered as a criterion for inclusion in the study the absence of missing data for any of the variables analyzed.

The dependent (explained) variable studied was the outcome of the patients, a dichotomous variable referring to the evolution of the case (death or discharge). The independent (explanatory) variable of greatest interest, analyzed uni and multivariately, was the presence or abscende of previous chronic neurological disease. The secondary independent variables, used only in the multivariate variable, were age, gender, and the presence or absence of each of the following: chronic heart disease, chronic hematologic disease, asthma, diabetes, chronic lung disease, chronic immunodepression, chronic kidney disease, and diabetes.

Initially, a descriptive analysis of the data was performed, calculating the proportion of patients with and without neurological disease, as well as the rates of occurrence of each outcome (death or discharge) globally and in relation to the presence or absence of chronic neurological disease. Next, univariate analysis was performed comparing these rates by calculating the risk ratio and Pearson's chi-square $\left(\chi^{2}\right)$ test. Finally, in order to verify the independence of the risk ratio observed in relation to the secondary explanatory variables, we performed a multivariate analysis using Poisson Regression.

For the statistical tests, a 95\% confidence interval (CI) was adopted and a p-value was considered significant when less than 0.05 . The analysis was performed by implementing the SciPy library (11).

Since the data used were collected in a public repository and were completely anonymized, it was not necessary to be assessed by the Research Ethics Committee. Thus, this research was performed in full compliance with ethical regulations (12).

\section{RESULTS}

The present study analyzed data from 87,871 patients, of whom 6,099 (6.9\%) had chronic neurological disease and 81,772 (93.1\%) did not. The numbers and rates of deaths stratified by the presence or absence of chronic neurological disease are presented in table 1 . The risk ratio (RR) for the outcome of death between patients with and without chronic neurological disease was $1.23(95 \%$ $\mathrm{CI}=1.2-1.3 ; \mathrm{p}$-value $<0.001)$.

TABLE 1. Mortality in patients with and without chronic neurological disease hospitalized for COVID-19

\begin{tabular}{|l|l|l|l|l|}
\hline \multirow{3}{*}{ Outcome } & \multicolumn{4}{|c|}{ Neurological disease } \\
\cline { 2 - 5 } & \multicolumn{2}{|c|}{ Present } & \multicolumn{2}{c|}{ Abscent } \\
\cline { 2 - 5 } & \multicolumn{1}{|c|}{ N } & \multicolumn{1}{c|}{ N } & \% \\
\hline Death event & 3171 & 52,0 & 34568 & 42,3 \\
\hline Discharge & 2928 & 48,0 & 47204 & 57,7 \\
\hline Total & $\mathbf{6 0 9 9}$ & 100,0 & $\mathbf{8 1 7 7 2}$ & $\mathbf{1 0 0 , 0}$ \\
\hline
\end{tabular}

Data source: SARS 2021 Severe Acute Respiratory Syndrome Database

Poisson Regression aimed to identify the independence of the association between the presence of chronic neurological disease and higher risk of death in relation to age, gender and other chronic diseases. The RR and $p$-value values from this multivariate analysis are shown in table 2.

\section{DISCUSSION}

It can be observed from the results described that presence of previous chronic neurological diseases is associated, with statistical significance and independently of gender, age, and other chronic diseases analyzed, with higher mortality in hospitalized cases of COVID-19. This finding of the present study, expressed in the $23 \%$ increase in the mortality rate of patients with chronic neurological diseases compared to those without, is consistent with the observation in a cohort study of 576 patients that chronic neurological diseases are an independent predictor of death in patients with COVID-19 (13).

It should be noted, however, that it is possible that this association is not specifically associated 
TABLE 2. Multivariate analysis by Poisson Regression for association with mortality in patients hospitalized for COVID-19

\begin{tabular}{|c|c|c|c|}
\hline Variable & Death RR & $95 \% \mathrm{Cl}$ & $\mathrm{p}$-value \\
\hline \multicolumn{4}{|l|}{ Age group } \\
\hline 0 to 4 years & 1 & - & - \\
\hline 5 to 19 years old & 1.07 & $0.78-1.45$ & 0.674 \\
\hline 20 to 39 years old & 3.18 & $2.54-3.99$ & $<0.001$ \\
\hline 40 to 59 years old & 4.35 & $3.48-5.45$ & $<0.001$ \\
\hline 60 to 79 years old & 6.41 & $5.13-8.02$ & $<0.001$ \\
\hline 80 or older & 8.06 & $6.45-10.09$ & $<0.001$ \\
\hline \multicolumn{4}{|l|}{ Sex } \\
\hline Female & 1 & - & - \\
\hline Male & 1.08 & $1.07-1.10$ & $<0.001$ \\
\hline \multicolumn{4}{|l|}{ Neurological disease } \\
\hline No & 1 & - & - \\
\hline Yes & 1.14 & 1.11-1.17 & $<0.001$ \\
\hline \multicolumn{4}{|l|}{ Cardiopathy } \\
\hline No & 1 & - & - \\
\hline Yes & 1.02 & $1.01-1.04$ & 0.002 \\
\hline \multicolumn{4}{|l|}{ Hematological disease } \\
\hline No & 1 & - & - \\
\hline Yes & 1.07 & $1.00-1.14$ & 0.034 \\
\hline \multicolumn{4}{|l|}{ Asthma } \\
\hline No & 1 & - & - \\
\hline Yes & 0.76 & $0.73-0.80$ & $<0.001$ \\
\hline \multicolumn{4}{|l|}{ Diabetes } \\
\hline No & 1 & - & - \\
\hline Yes & 1.11 & $1.09-1.13$ & $<0.001$ \\
\hline \multicolumn{4}{|l|}{ Pneumopathy } \\
\hline No & 1 & - & - \\
\hline Yes & 1.09 & $1.07-1.12$ & $<0.001$ \\
\hline \multicolumn{4}{|l|}{ Immunodepression } \\
\hline No & 1 & - & - \\
\hline Yes & 1.22 & $1.18-1.26$ & $<0.001$ \\
\hline \multicolumn{4}{|l|}{ Kidney Disease } \\
\hline No & 1 & - & - \\
\hline Yes & 1.22 & $1.19-1.25$ & $<0.001$ \\
\hline \multicolumn{4}{|l|}{ Obesity } \\
\hline No & 1 & - & - \\
\hline Yes & 1.26 & $1.23-1.28$ & $<0.001$ \\
\hline
\end{tabular}

Data source: SARS 2021 Severe Acute Respiratory Syndrome Database

with the pathophysiological process triggered by SARS-CoV-2 infection, but rather with the lower health reserve and fragility of these patients when facing diseases and hospitalization in general. The observation that individuals affected by neurological diseases are at greater risk of death event when hospitalized contributes to corroborate this hypothesis (14). The consideration that other chronic diseases analyzed by Poisson Regression also had a statistically significant association with increased risk of death in hospitalized patients by COVID-19 also points in this direction.

Still, it is important to further investigate and better understand the already demonstrated effects that SARS-CoV-2 is capable of exerting on the central nervous system. Like other organs, the brain expresses human angiotensin-converting enzyme 2 (hACE2) protein, a binding site for the said virus, and such viral agent can trigger in the host an immune response involving increased cytokine release, tissue damage, and high neuro-susceptibility $(8,15)$.

A great potential of our study was the availability of data from a large number of patients. However, the absence of more specific clinical information (such as specific neurological involvement and more details on the clinical, imaging, and laboratory progression of the data) is a limitation. Another limitation is that these data come from reports in which the completion of all interrogated fields was not mandatory, which may have generated some bias in the selection of those included in the analysis (for example, by a higher percentage exclusion of patients without neurological disease than with COVID-19 hospitalizations, if the absence of an involvement was a trigger for completion of the corresponding field). Nevertheless, it provides valuable insights with great statistical robustness and it reinforces observations previously recorded in the literature.

\section{CONCLUSIONS}

Chronic neurological diseases have been observed as an independent risk factor for mortality in patients hospitalized for COVID-19, such that this constitutes relevant clinical data in the management and diagnostic evaluation of these patients. However, studies are needed to better characterize this association, especially with regard to investigating whether there is a difference in risk among different chronic neurological diseases and whether this increased risk differs from the increased risk already observed in the presence of chronic neurological disease in hospitalized patients in general (regardless of the cause of hospitalization). 


\section{REFERENCES}

1. Guan WJ, Ni ZY, Hu Y, Lian W, Ou C, He J, et al. Clinical characteristics of Coronavirus Disease 2019 in China. N Engl J Med. 2020;382:1708-1720.

2. Zhou F, Yu T, Du R, Fan G, Liu Y, Liu Z, et al. Clinical course and risk factors for mortality of adult inpatients with COVID-19 in Wuhan, China: a retrospective study. Lancet. 2020;395:1054-1062.

3. Grasselli G, Greco M, Zanella A, Albano G, Antonelli M, et al.; COVID-19 Lombardy ICU Network. Risk Factors Associated With Mortality Among Patients With COVID-19 in Intensive Care Units in Lombardy, Italy. JAMA Intern Med. 2020;180(10):1345-1355.

4. Meshkat S, Salimi A, Joshaghanian A, Sedighi S, Sedighi S, Aghamollaii V. Chronic neurological diseases and COVID-19: Associations and considerations. Translational Neuroscience. 2020;11(1):294-301.

5. Espinosa PS, Rizvi Z, Sharma P, Hindi F, Filatov A. Neurological complications of coronavirus disease (COVID-19): encephalopathy, MRI brain and cerebrospinal fluid findings: case 2. Cureus. 2020;12(5):e7930.

6. Kanwar D, Baig AM, Wasay M. Neurological manifestations of COVID-19. J Pak Med Assoc. 2020;70(5):101-103.

7. Asadi-Pooya AA, Simani L. Central nervous system manifestations of COVID-19: a systematic review. J Neurol Sci. 2020;413:e116832.

8. Assari S. COVID-19 Pandemic and Neurological Disease: A Critical Review of the Existing Literature. Hosp Pract Res. 2020;5(3):81-86.

9. Ministry of Health (BR). Secretariat of Health Surveillance. Ficha de registro individual: cases of severe acute respiratory syndrome hospitalized [Internet]. Brasilia: Ministry of Health; 2021 [Accessed 08.04.2021]. Avalaible at: https://opendatasus.saude.gov.br/ dataset/9f76e80f-a2f1-4662-9e37-71084eae23e3/resource/ bd725e4c-c869-400f-a479-9b544c2812b4/download/ficha-sragfinal-27.07.2020_final.pdf.

10. [DATABASE] Informatics Department of the Brazilian Unified Health System, 2021. SARS 2021 Severe Acute Respiratory Syndrome Database. [Accessed 04.04.2021]. Available at: https://opendatasus. saude.gov.br/dataset/bd-srag-2021.

11. Virtanen P, Gommers R, Oliphant TE, Haberland M, et al.; SciPy 1.0 Contributors. (2020) SciPy 1.0: Fundamental Algorithms for Scientific Computing in Python. Nature Methods. 2020; 17(3):261-272.

12. National Health Council (BR), 2016. Resolution 510, of April 7, 2016. [Accessed 08.04.2021]. Available at: http://conselho.saude.gov.br/ resolucoes/2016/Res0510.pdf.

13. García-Azorín D, Martínez-Pías E, Trigo J, et al. Neurological Comorbidity Is a Predictor of Death in COVID-19 Disease: A Cohort Study on 576 Patients. Front. Neurol. 2020;11:e781.

14. Schwartz N, Sakhinini, Bisharat N. Predictive modeling of inpatient mortality in departments of internal medicine. Intern Emerg Med. 2018;13:205-211.

15. Das G, Mukherjee N, Ghosh S. Neurological Insights of COVID-19 Pandemic. ACS Chem Neurosci. 2020;11(9):1206-1209. 